\title{
ANÁLISE DO DESEMPENHO DA INOVAÇÃO REGIONAL - UM ESTUDO DE CASO NA INDÚSTRIA
}

\author{
Claudio Alcides Jacoski \\ Doutor em Engenharia de Produção pela Universidade Federal de Santa Catarina - UFSC \\ Professor do Programa de Pós-Graduação em Tecnologia e Gestão da Inovação da Universidade \\ Comunitária Regional de Chapecó - Unochapecó \\ claudio@unochapeco.edu.br (Brasil)
}

\section{Caroline Dallacorte}

Engenheira de Alimentos pela Universidade Comunitária da Região de Chapecó - Unochapecó

Núcleo de Gestão e Competitividade Empresarial da Universidade Comunitária da Região de Chapecó

- Unochapecó

carold@unochapeco.edu.br (Brasil)

\section{Beno Nicolau Bieger}

Doutor em Engenharia Florestal pela Universidade Federal do Paraná - UFPR

Professor do Programa de Pós-Graduação em Tecnologia e Gestão da Inovação da Universidade Comunitária da Região de Chapecó - Unochapecó

beno@unochapeco.edu.br (Brasil)

\section{Moacir Francisco Deimling}

Doutor em Engenharia de Produção pela Universidade Federal do Rio Grande do Sul - UFRGS Professor da Universidade Federal da Fronteira Sul - UFFS moacir.deimling@uffs.edu.br (Brasil)

\section{RESUMO}

Este artigo trata da análise de desempenho de inovação regional, realizada com o intuito de, a partir de uma adaptação metodológica, investigar o nível de inovação tecnológica nas indústrias de uma região no período de quatro anos e a relação com o desenvolvimento regional. Desenvolveu-se um método baseado no PINTEC/IBGE associado ao Manual de Oslo. Uma pesquisa semiestruturada foi aplicada em 54 indústrias de diversos setores que mais caracterizam o aporte ao desenvolvimento dessa região. Os resultados mostraram que apesar dos inúmeros obstáculos enfrentados as indústrias estão obtendo um bom nível de atividade inovadora nos seus produtos e processos, melhorando assim a competitividade no mercado e contribuindo para o desenvolvimento da região.

Palavras-chave: Inovação tecnológica; Gestão da inovação; Manual de Oslo; Indústrias. 


\section{INTRODUÇÃO}

A inovação caracteriza-se como a descoberta, a busca, experimentação e desenvolvimento de novos produtos, processos e também novas técnicas organizacionais. É um procedimento interativo, em que existe a contribuição tanto de agentes econômicos como sociais, incluindo diferentes tipos de informações e conhecimentos que obrigatoriamente têm uma relação direta com os agentes regionais. A inovação tecnológica hoje faz parte do mundo globalizado, sendo utilizada como uma forma estratégica de competição entre empresas.

Hitt et al (2002) defendem a ideia de que a inovação impulsiona o sucesso competitivo das empresas não somente com a criação de novos produtos, mas também através de mudanças na sua estrutura organizacional.

Para ter sucesso ao incluir um novo sistema/produto no mercado de trabalho são necessários alguns fatores integrados, como tecnologia, ambiente externo, pessoas e a própria organização, que harmonizados podem produzir desenvolvimento no seu mais amplo aspecto.

Para o Movimento Brasil Competitivo (2008), a inovação no meio empresarial é a exploração de novas ideias para alavancagem dos negócios, criando vantagens competitivas, gerando capital para a empresa e consequentemente sucesso no mercado. Ela pode ser realizada pela empresa, individualmente ou em parceria com outras instituições, ou também adaptando ideias de outras empresas nacionais e estrangeiras.

Conhecer o processo de gestão da inovação como um dos elementos que contribui para o desenvolvimento regional foi o foco deste projeto que culmina com a apresentação dos resultados de uma pesquisa realizada em indústrias da cidade de Chapecó e região Oeste Catarinense, através da aplicação de um questionário com base na Pesquisa de Inovação Tecnológica - PINTEC, realizada pelo Instituto Brasileiro de Geografia e Estatística - IBGE e do Manual de Oslo. O objetivo foi avaliar o nível de inovação tecnológica e identificar as dificuldades que as indústrias enfrentam em relação à inovação, bem como sua relação com o desenvolvimento da região.

\section{A INOVAÇÃO E O PROCESSO DE EMPREENDER}

Os primeiros estudos a abordar a inovação no processo empreendedor foram de Schumpeter, ao tratar da "destruição criadora", quando o empreendedor destrói a ordem econômica existente com a introdução de algo novo. O conceito de inovação proposto por Schumpeter (1982) compreende cinco 
tipos: (1) introdução de um novo bem; (2) introdução de um novo método de produção; (3) abertura de um novo mercado; (4) conquista de uma nova fonte de oferta de matérias-primas ou de bens semimanufaturados; e (5) o estabelecimento de uma nova organização de qualquer indústria. Vale destacar que a visão schumpeteriana de inovação está predominantemente associada à inovação radical, ou seja, o autor não considera como inovação as melhorias técnicas realizadas em novos produtos ou processos após sua introdução no mercado (HAGEDOORN, 1994).

Depois de Schumpeter, o conceito de inovação passou por um processo evolutivo. Segundo a Organização para Cooperação e Desenvolvimento Econômico (OCDE, 1992), a inovação é caracterizada pela transformação de uma ideia num novo produto, num processo operacional para a indústria ou para o comércio ou num novo método social.

Do Manual Frascati ao Manual de Oslo e suas atualizações, a definição de inovação foi evoluindo. Inovações tecnológicas em produtos e processos (TPP) compreendem a implantação de produtos e processos tecnologicamente novos e/ou substancialmente melhorados. Uma inovação TPP é considerada implantada se for introduzida no mercado (inovação de produto) ou usada no processo de produção (inovação de processo). Implantar uma inovação TPP envolve uma série de atividades científicas, tecnológicas, organizacionais, financeiras e comerciais. Uma empresa inovadora em TPP é uma empresa que tenha implantado produtos ou processos tecnologicamente novos ou com substancial melhoria tecnológica durante um determinado período.

A primeira edição do Manual de Oslo foi publicada em 1992 e serviu de base para o Community Innovation Survey (CIS) organizado pela União Europeia. A segunda edição, de 1997, atualizou a estrutura de conceitos, definições e metodologia para incorporar experiências de pesquisas, ampliando a compreensão do processo de inovação. As duas primeiras edições usaram definição de inovação tecnológica de produtos e de processo (TPP). A terceira edição, de 2005, introduz outros dois tipos de inovação: organizacional e de marketing.

Qualquer forma de inovação envolve uma série de esforços: a inovatividade tecnológica, por exemplo, envolve primeiramente esforço de pesquisa e de engenharia, focado no desenvolvimento de novos produtos e processos; a inovatividade em produto e mercado inclui pesquisa de mercado, design de produto, e inovação em publicidade e promoção; a inovatividade administrativa refere-se a novidades em sistemas gerenciais, técnicas de controle e estrutura organizacional (DESS et al, 2005).

As empresas inovam ou para defender suas posições competitivas ou em busca de vantagem competitiva. Uma empresa pode ter uma abordagem reativa e inovar para evitar perder participação de mercado para um concorrente inovador. Pode, também, ter uma abordagem preventiva e buscar uma 
posição estratégica no mercado em relação a seus competidores, desenvolvendo e tentando impor, por exemplo, padrões técnicos mais altos para os produtos que produz (OCDE, 2005).

Segundo Tether (2003), as empresas que inovam são dotadas de rotinas e processos sistemáticos focalizados nas habilidades de aprender e adaptar. Essas empresas são comprometidas com a prática de melhorias que possam culminar em novos produtos ou novos processos. Nesse sentido, Tether (2003, p. 10) menciona que as empresas que são inovadoras "tendem a ter um padrão instruído e estável de atividade coletiva pela qual a organização gera e modifica sistematicamente suas rotinas operacionais em busca de melhor efetividade."

A inovação e o sucesso da inovação dependem em larga escala do comportamento do consumidor. Traill (1997) argumenta que mudanças no padrão de consumo muito frequentemente determinam a natureza da inovação que ocorre na indústria.

As necessidades e desejos dos consumidores são a fonte lógica para buscar ideias de novos produtos. Muitas das melhores ideias surgem quando se pede aos consumidores para descreverem seus problemas em relação aos produtos atuais (KOTLER, 2000).

Segundo Kotler (2000), um cliente altamente satisfeito ou "encantado" com a organização, tem um valor dez vezes maior do que um cliente apenas satisfeito. Esse encantamento do cliente somente é atingido através da oferta constante de produtos e/ou serviços diferenciados em relação aos produtos e/ou serviços oferecidos pelos concorrentes, ou seja, se obtém vantagens competitivas através da inovação contínua.

De acordo com Ferraz (1995), o processo de inovação vem sendo um dos indicadores mais utilizados para avaliar a competitividade, uma vez que seus resultados se encontram vinculados à capacidade de acompanhar as mudanças e o desenvolvimento do mercado, bem como a criação e ocupação de novos mercados - processo cada vez mais dinâmico.

Na visão de Sbragia (2001), o investimento em pesquisa e desenvolvimento (P\&D) gera inovações que por consequência trazem maiores lucros e competitividade para as empresas. Analisando a área de P\&D das organizações, Stefanovitz e Nagano (2005) apresentam situações nas quais as empresas de alta tecnologia também podem obter uma maior lucratividade comercializando os produtos, processos ou serviços gerados a partir das atividades de $\mathrm{P} \& \mathrm{D}$ com terceiros, realizando uma forma de licenciamento destas inovações criadas pela empresa. Porém, dentre as atividades inovativas, a de P\&D é a que apresenta as maiores incertezas.

Apesar de todas as oportunidades de sucesso vindas com a inovação, há também um aumento de risco. No caso de grandes organizações, o custo de eventuais erros decorrentes de experiências inovadoras malsucedidas é amplamente compensado pelos casos de sucesso. Nas micro e pequenas

Revista de Administração e Inovação, São Paulo, v. 11, n.2, p. 71-88, abr./jun. 2014. 
empresas (MPE), entretanto, uma simples troca de cor na embalagem pode levar a resultados que a empresa não tem condições de suportar (BACHMANN et al, 2008).

\section{A INOVAÇÃO EM MICRO, PEQUENAS E MÉDIAS EMPRESAS}

As micro, pequenas e médias empresas (MPMEs) vêm sendo há muito tempo alvo de atenção de analistas econômicos devido a seu potencial de geração de renda e de emprego. No pós-fordismo, essa atenção se intensifica à medida que os atributos de flexibilidade e rapidez de adaptação às demandas do mercado, características de muitas MPMEs, são valorizadas (LA ROVERE, 1999).

Uma nação é forte quando tem um grande número de pequenas e médias empresas permeando todos os setores produtivos e difundidas em todos os segmentos da economia e em todas as regiões. A estratégia de inovação via agregação de novos processos e padrões tecnológicos e a busca de maior flexibilidade constituem caminho alternativo adotado para as pequenas e médias empresas se inserirem competitivamente num processo de demandas mutantes, instáveis e diferenciadas (CARON, 2004).

Como observado por Rothwell e Dodgson (1993), tanto as MPMEs como as grandes empresas têm vantagens para gerar e adotar inovações. Enquanto as grandes empresas têm vantagens materiais, devido à sua maior capacidade de $\mathrm{P} \& \mathrm{D}$, as pequenas e médias empresas têm vantagens comportamentais relacionadas à maior flexibilidade e capacidade de adaptação a mudanças no mercado.

Cabe observar também que as MPMEs podem não ter consciência dos possíveis ganhos de competitividade trazidos pelas inovações. A maioria dessas empresas gera ou adota inovações apenas quando percebem claramente as oportunidades de negócio ligadas à inovação ou quando estão sob pressão de clientes e/ou fornecedores (GAGNON e TOULOUSE, 1996).

Entretanto, as pequenas empresas não têm necessariamente um potencial inovador maior do que as grandes, pois têm menor acesso a informações tecnológicas e, portanto, podem ser menos propensas à inovação (OECD, 1995).

Segundo Caron (2004), as principais dificuldades enfrentadas pelas pequenas e médias empresas para inovar são, em ordem de importância, a falta de:

- recursos para investir em inovação;

- acesso a financiamento para inovação;

- informações sobre entidades de apoio à inovação tecnológica;

- pessoal capacitado;

- máquinas e equipamentos;

Revista de Administração e Inovação, São Paulo, v. 11, n.2, p. 71-88, abr./jun. 2014. 
- informações sobre mudanças tecnológicas;

- confiança em parcerias e alianças para inovação tecnológica;

- informações sobre mercados.

\section{FERRAMENTAS PARA AVALIAÇÃO DA INOVAÇÃO}

\subsection{Manual de Oslo}

O Manual de Oslo, desenvolvido conjuntamente pelo Eurostat e a OCDE, constitui parte de uma família de manuais dedicada à mensuração e interpretação de dados relacionados a ciência, tecnologia e inovação. Esse material compreende manuais, diretrizes e guias sobre P\&D (Manual Frascati), indicadores de globalização, patentes, a sociedade da informação, recursos humanos em ciência e tecnologia (C\&T) (Manual Canberra) e estatísticas de biotecnologia.

O objetivo do Manual é oferecer diretrizes para a coleta e a interpretação de dados sobre inovação. Os dados sobre inovação podem ser utilizados para diversas finalidades e o Manual foi concebido para acomodar esses usos. Uma razão para a coleta de dados de inovação é compreender melhor essas atividades e sua relação com o crescimento econômico.

O Manual visa à coleta de dados sobre inovação no âmbito da firma. Ele não trata de mudanças amplas na indústria ou na economia, tais como a emergência de um mercado novo, o desenvolvimento de uma nova fonte de matérias-primas ou de bens semimanufaturados ou a reorganização da indústria. Contudo, é possível, em alguns casos, estimar mudanças mais abrangentes na indústria e na economia por meio da agregação de dados de firmas individuais (OCDE, 2005).

\subsection{PINTEC}

A Pesquisa de Inovação (Pintec) é realizada pelo Instituto Brasileiro de Geografia e Estatística (IBGE), com o apoio da Financiadora de Estudos e Projetos (Finep) e do Ministério da Ciência, Tecnologia e Inovação (MCTI).

A Pintec tem como principal objetivo conhecer as atividades inovativas desenvolvidas nas empresas industriais, de telecomunicações, de atividades de informática e serviços relacionados e de pesquisa e desenvolvimento, de modo a acompanhar sua evolução no tempo. 
Os resultados agregados da pesquisa permitem às empresas avaliar o seu desempenho em relação às médias setoriais e às entidades de classe, analisar as características setoriais da inovação e aos governos, desenvolver e avaliar políticas nacionais e regionais (IBGE, 2012).

\section{MÉTODOS E PROCESSOS}

Nesta pesquisa foi implementada uma associação dos dois métodos conhecidos, Pintec e Manual de Oslo, transformando-os em uma pesquisa semi-estruturada. Baseado na pesquisa de inovação do Pintec e nos conceitos de inovação tecnológica do Manual de Oslo, se buscou avaliar o nível tecnológico das indústrias de Chapecó e da região oeste catarinense nos últimos quatro anos. Foi elaborado um questionário, aplicado em forma de pesquisa semiestruturada, contendo 70 quesitos que foram utilizados para investigação em 54 indústrias de diversos setores.

\section{ANÁLISE DOS RESULTADOS E DISCUSSÃO}

Das 54 indústrias pesquisadas, 49 estão localizadas na cidade de Chapecó e cinco são empresas da região. Dividem-se em diversos setores de atividades industriais como pode ser observado na tabela abaixo.

Tabela 1: Setor de atividades das indústrias

\begin{tabular}{|l|l|l|}
\hline Setor de atividade & Freq. & \multicolumn{1}{c|}{} \\
\hline Alimentos & 21 & $38,9 \%$ \\
\hline Metal Mecânica & 12 & $22,2 \%$ \\
\hline Moveleira & 7 & $13,0 \%$ \\
\hline Plásticos & 6 & $11,1 \%$ \\
\hline Outros, qual? & 5 & $9,3 \%$ \\
\hline Têxtil & 3 & $5,6 \%$ \\
\hline TOTAL & $\mathbf{5 4}$ & $\mathbf{1 0 0 \%}$ \\
\hline
\end{tabular}




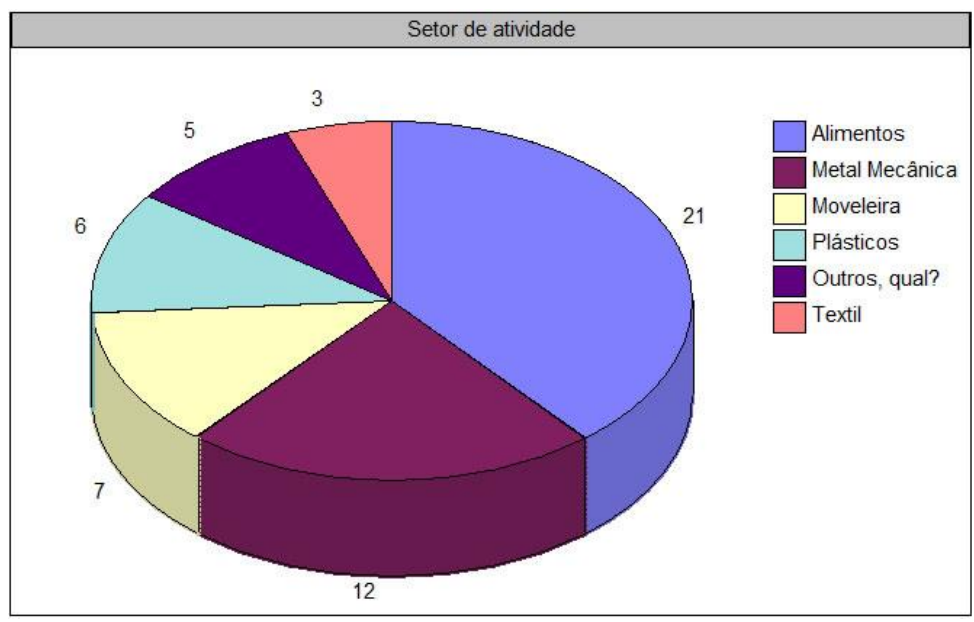

Gráfico 1: Setor de atividade das indústrias

As inovações de produto compreendem produtos novos e produtos aperfeiçoados. Produto novo (bem ou serviço) é um produto cujas características fundamentais (especificações técnicas, matériasprimas, componentes, software incorporado, funções ou usos pretendidos) diferem significativamente de todos os produtos previamente produzidos pela empresa.

Significativo aperfeiçoamento de produto (bem ou serviço) se refere a um produto previamente existente, cujo desempenho foi substancialmente incrementado ou aperfeiçoado, através de mudanças nas matérias-primas, componentes ou em outras características que melhoram sua performance (IBGE, 2012).

Nos últimos quatro anos, nas empresas consultadas, foram implantados 19 produtos novos e 28 aperfeiçoados. Em termos de mercado, além dos produtos implantados no mercado nacional, constatou-se a implantação de sete produtos para o mercado internacional.

Desses produtos, 31 foram desenvolvidos pela própria empresa, 13 são resultado de parcerias com outras empresas, duas empresas estabeleceram parcerias com instituições e uma utilizou serviço de contratação de outras empresas.

Inovação de processo se refere à implementação de um novo ou substancialmente aperfeiçoado método de produção ou de entrega de produtos (bens ou serviços). Envolve também mudanças significativas em técnicas, equipamentos e/ou softwares empregados em atividades de apoio à produção.

Métodos de produção novos ou significativamente aperfeiçoados, na indústria, envolvem mudanças nas técnicas, máquinas, equipamentos ou softwares usados nos processos de transformação de insumos em produtos. Nos serviços, métodos de produção novos ou significativamente aperfeiçoados podem envolver mudanças nos equipamentos ou softwares utilizados, como também nos procedimentos ou técnicas que são empregados para criação e fornecimento dos serviços.

Revista de Administração e Inovação, São Paulo, v. 11, n.2, p. 71-88, abr./jun. 2014. 
Métodos de entrega novos ou significativamente aperfeiçoados dizem respeito a mudanças na logística da empresa, que engloba equipamentos, softwares e técnicas de suprimento de insumos, estocagem, acondicionamento, movimentação e entrega de bens ou serviços (IBGE, 2012).

Das 54 indústrias avaliadas, 44 tiveram métodos de processo de fabricação, produção ou prestação de serviços novo ou aperfeiçoado, de tal maneira que 11 processos podem ser considerados como novos. No âmbito da possibilidade de utilização, 32 eram novos e/ou aperfeiçoados para a empresa, 10 para o mercado nacional e dois para o mercado internacional.

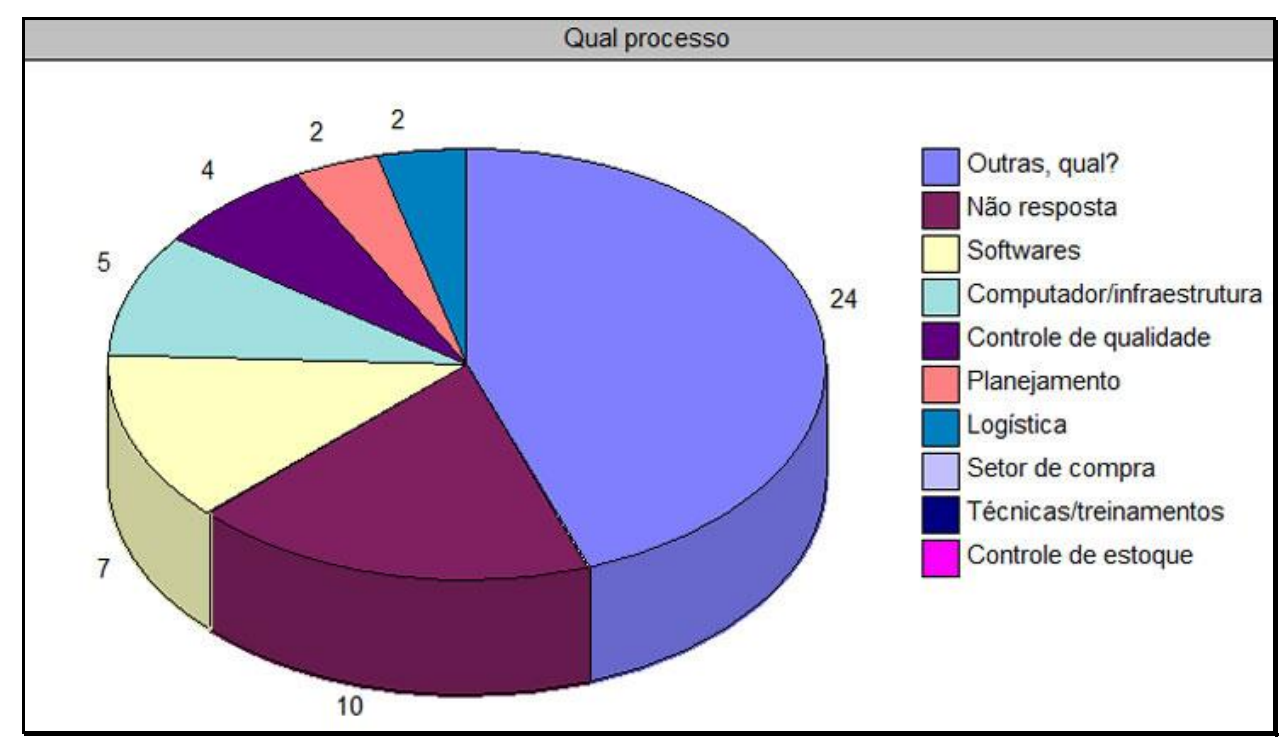

Gráfico 2: Tipos de processos novos e/ou aperfeiçoados na indústria

Para 28 indústrias, esse processo teve importância alta para o desenvolvimento. Em 15 indústrias a importância foi média, em uma indústria a importância foi considerada baixa e 10 das indústrias entrevistadas não responderam.

Com relação às empresas que implantaram as inovações de processos na indústria, 27 foram realizados pela empresa, 11 em parceria com outras empresas, 10 através da contratação de outras empresas, três com parceria entre instituições, três com outra empresa do grupo.

Pesquisa e Desenvolvimento (P\&D) podem ser considerados um processo de redução de incerteza ou aprendizado, associados a esforços paralelos para lidar com a incerteza. O custo de um projeto de desenvolvimento em particular depende do tamanho e da complexidade do produto a ser desenvolvido; do grau de avanço do desempenho em que se baseia; do estoque de conhecimentos básicos, materiais e componentes e do tempo de desenvolvimento (MANSFIELD,1978).

Com relação a $\mathrm{P} \& \mathrm{D}$, apenas 16 indústrias avaliadas possuem essa atividade. Dessas 12 possuem P\&D interna. As demais não possuem ou as atividades são realizadas externamente. 
Tabela 2: P\&D realizada de forma Interna ou Externa

\begin{tabular}{|l|l|l|}
\hline P\&D interna ou externa & Freq. & \% \\
\hline Não possui & $\mathbf{3 5}$ & $\mathbf{6 4 , 8 \%}$ \\
\hline Interna & $\mathbf{1 2}$ & $\mathbf{2 2 , 2 \%}$ \\
\hline Proprietários & $\mathbf{4}$ & $\mathbf{7 , 4 \%}$ \\
\hline Externa - Consultorias & $\mathbf{2}$ & $\mathbf{3 , 7 \%}$ \\
\hline Externa - Instituições & $\mathbf{1}$ & $\mathbf{1 , 9 \%}$ \\
\hline Externa - Empresas & $\mathbf{1}$ & $\mathbf{0 , 0 \%}$ \\
\hline TOTAL & $\mathbf{5 4}$ & $\mathbf{1 0 0 \%}$ \\
\hline
\end{tabular}

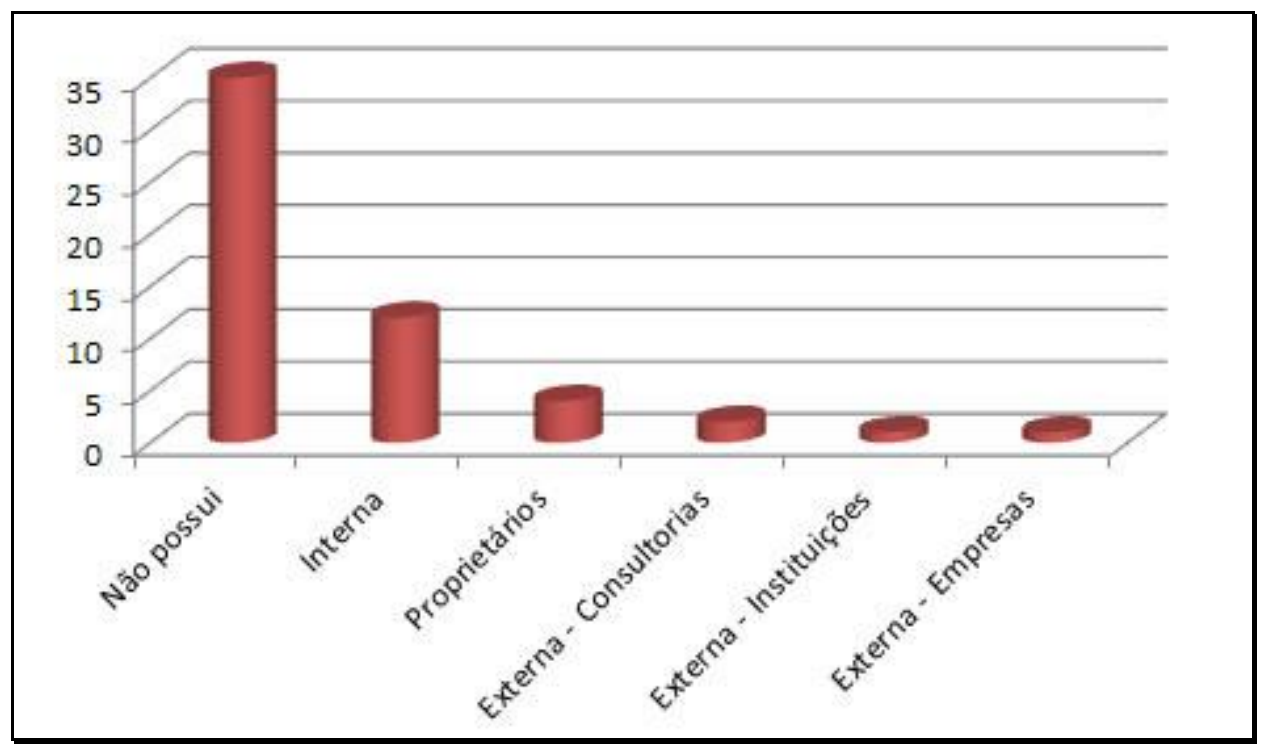

Gráfico 3: Forma de realização de P\&D

Das 54 indústrias analisadas 23 obtiveram atividade inovadora de $\mathrm{P} \& \mathrm{D}$ nos últimos quatro anos. Entre estas 13 delas consideraram a atividade de importância alta para o desenvolvimento da indústria.

Entre as indústrias que realizaram algum tipo de inovação, 25 afirmaram encontrar algum tipo de dificuldade ou obstáculo que tornou mais lenta ou inviabilizou a implementação de determinados projetos. Outras 16 informaram que a dificuldade ou obstáculo ocorreu em partes, 10 indústrias não encontraram problemas e 3 não souberam responder.

No Gráfico 4 são apresentados os motivos apontados pelas empresas que não desenvolveram atividade inovadora nos últimos quatro anos. 


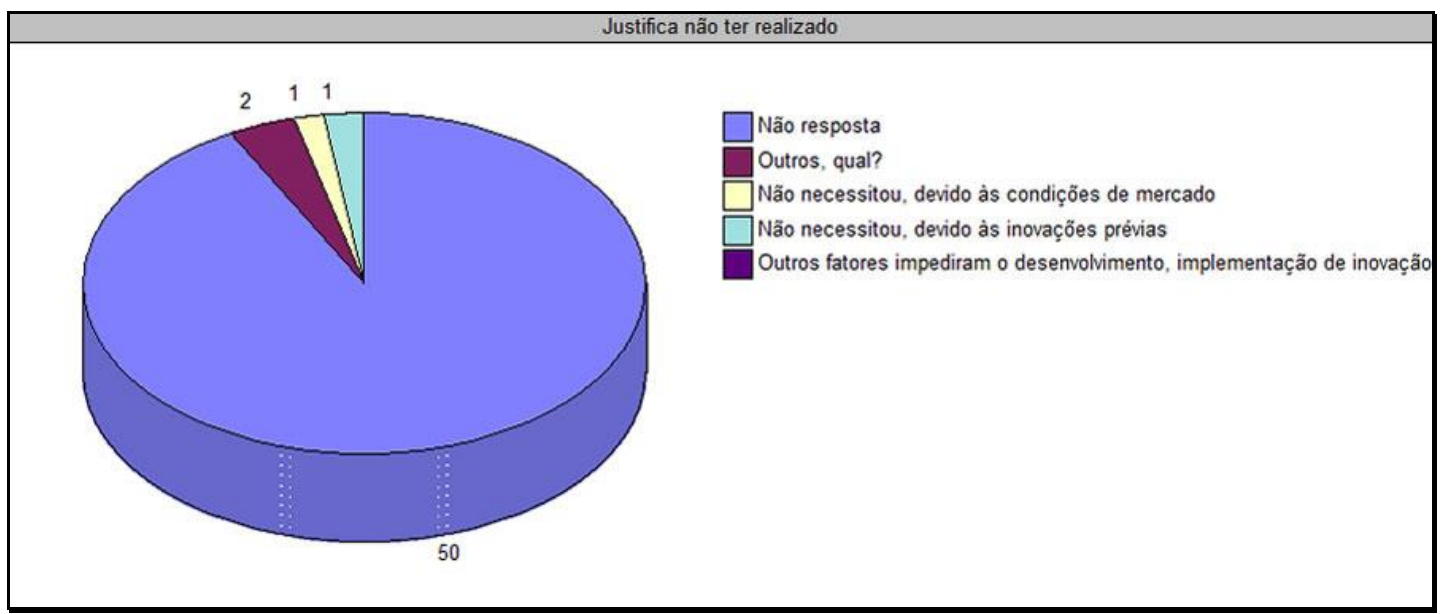

Gráfico 4: Razões pela qual as indústrias não realizaram atividade inovadora

O grau de importância dos fatores que prejudicaram as atividades inovadoras das indústrias, é descrito na Tabela 3, obtido com a indicação das indústrias entrevistadas durante a pesquisa.

Tabela 3: Fatores com grau de importância alto que prejudicaram a inovação das indústrias.

\begin{tabular}{|l|l|l|}
\hline Grau de importância: Alto & Freq. & \% \\
\hline Riscos econômicos excessivos & 19 & $\mathbf{3 5 , 2 \%}$ \\
\hline Elevados custos de inovação & $\mathbf{2 9}$ & $\mathbf{5 3 , 7 \%}$ \\
\hline Escassez de fontes apropriadas de financiamento & $\mathbf{1 7}$ & $\mathbf{3 1 , 5 \%}$ \\
\hline Rigidez organizacional & $\mathbf{1 3}$ & $\mathbf{2 4 , 1 \%}$ \\
\hline Falta de pessoal qualificado & $\mathbf{4 1}$ & $\mathbf{7 5 , 9 \%}$ \\
\hline Falta de informação sobre tecnologia & $\mathbf{1 4}$ & $\mathbf{2 5 , 9 \%}$ \\
\hline Escassa possibilidade de cooperação - empresas/instituições & $\mathbf{1 8}$ & $\mathbf{3 3 , 3 \%}$ \\
\hline Dificuldade para se adequar a padrões e normas & $\mathbf{1 9}$ & $\mathbf{3 5 , 2 \%}$ \\
\hline A fraca resposta dos consumidores quanto a novos produtos & $\mathbf{7}$ & $\mathbf{1 3 \%}$ \\
\hline Centralização da atividade inovadora em outra empresa & $\mathbf{4}$ & $\mathbf{7 , 4 \%}$ \\
\hline
\end{tabular}




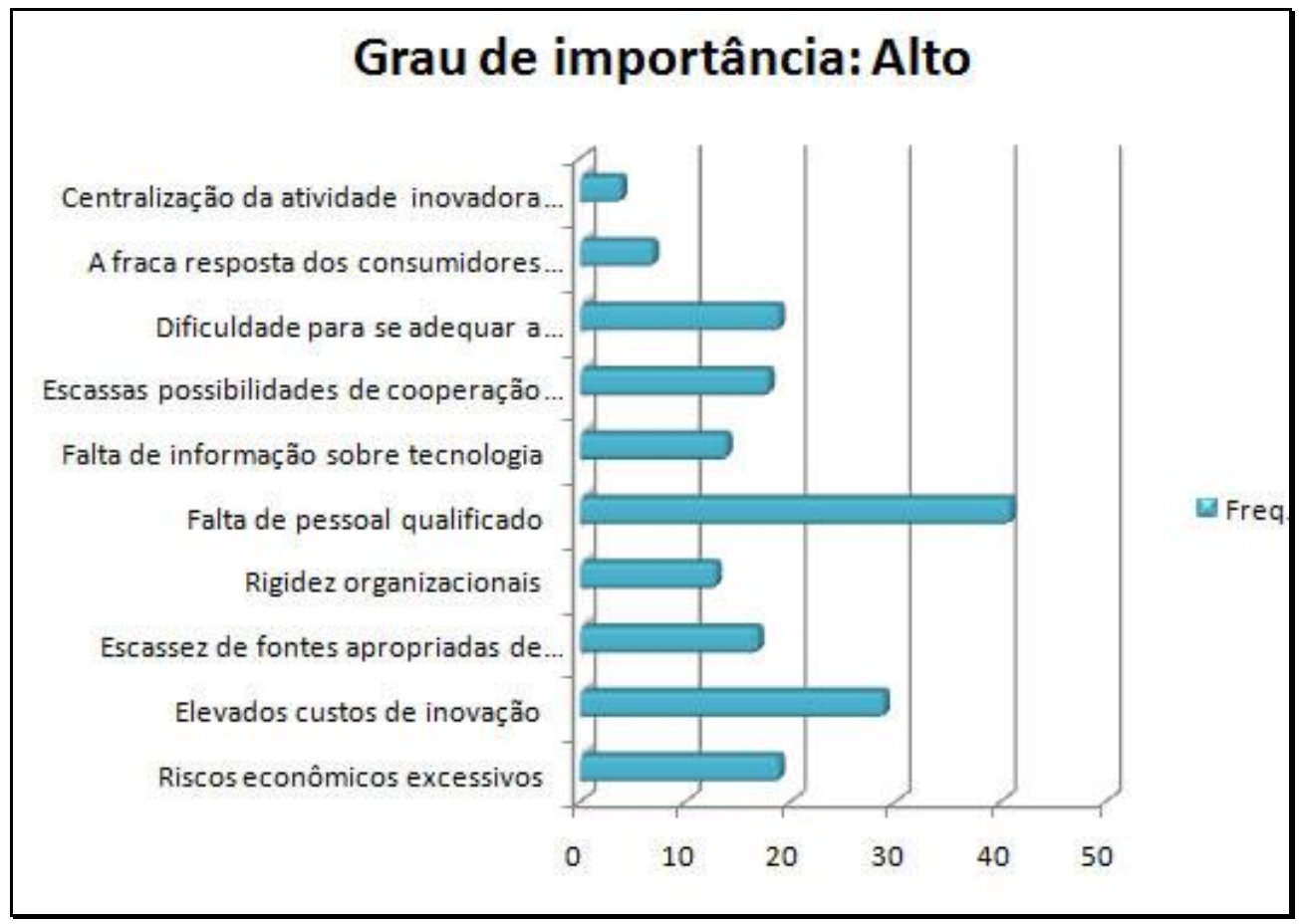

Gráfico 5: Fatores que prejudicaram a inovação das indústrias com grau de importância alto.

Tabela 4: Fatores com grau de importância médio que prejudicaram a inovação das indústrias. (Frequência e Porcentagem baseados no total de 54 indústrias).

\begin{tabular}{|l|l|l|}
\hline Grau de importância: Médio & Freq. & \% \\
\hline Riscos econômicos excessivos & $\mathbf{2 1}$ & $\mathbf{3 8 , 9 \%}$ \\
\hline Elevados custos de inovação & $\mathbf{1 8}$ & $\mathbf{3 3 , 3 \%}$ \\
\hline Escassez de fontes apropriadas de financiamento & $\mathbf{1 7}$ & $\mathbf{3 1 , 5 \%}$ \\
\hline Rigidez organizacionais & $\mathbf{2 3}$ & $\mathbf{4 2 , 6 \%}$ \\
\hline Falta de pessoal qualificado & $\mathbf{4}$ & $\mathbf{7 , 4 \%}$ \\
\hline Falta de informação sobre tecnologia & $\mathbf{2 4}$ & $\mathbf{4 4 , 4 \%}$ \\
\hline Escassas possibilidades de com & $\mathbf{1 6}$ & $\mathbf{2 9 , 6 \%}$ \\
\hline Dificuldade para se adequar a padrões, normas e & $\mathbf{1 9}$ & $\mathbf{3 5 , 2 \%}$ \\
\hline A fraca resposta dos consumidores quanto a novos produtos & $\mathbf{1 5}$ & $\mathbf{2 7 , 8 \%}$ \\
\hline Centralização da atividade inovadora em outra empresa do & $\mathbf{3}$ & $\mathbf{5 , 6 \%}$ \\
\hline
\end{tabular}




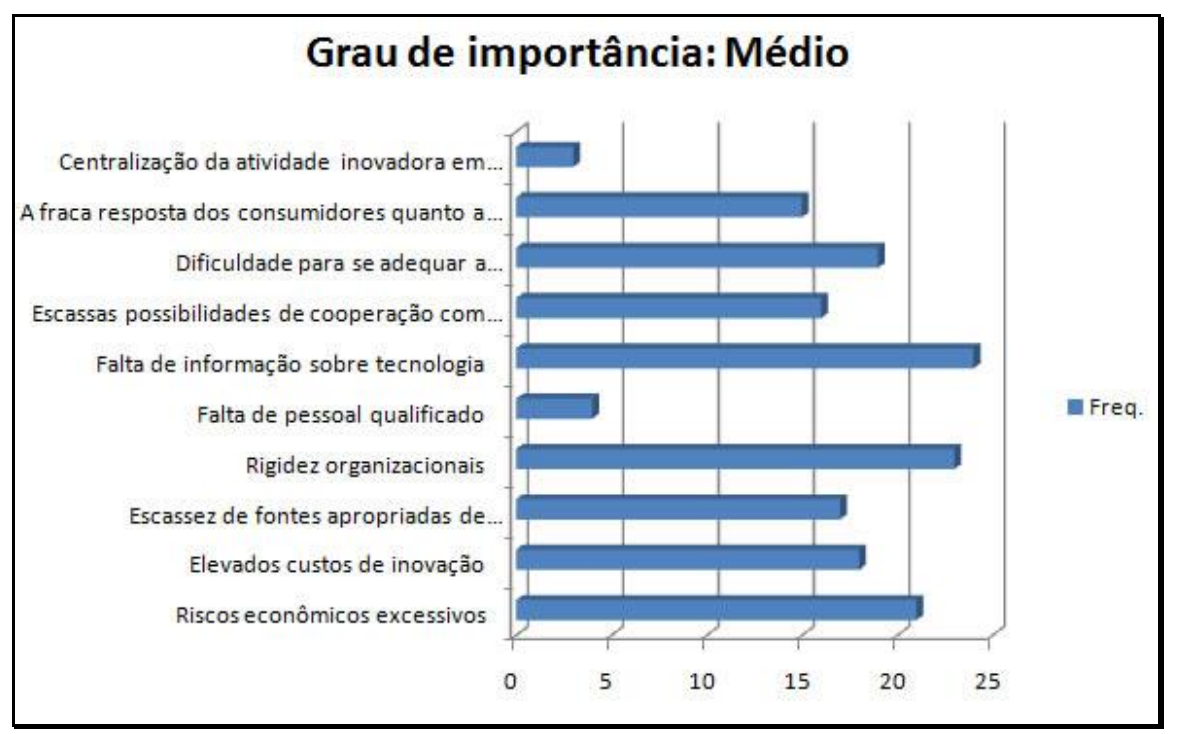

Gráfico 6: Fatores que prejudicaram a inovação das indústrias com grau de importância médio.

Tabela 5: Fatores que prejudicaram a inovação das indústrias com grau de importância baixo.

\begin{tabular}{|l|l|l|}
\hline Grau de importância: Baixo & Freq. & \% \\
\hline Riscos econômicos excessivos & $\mathbf{8}$ & $\mathbf{1 4 , 8 \%}$ \\
\hline Elevados custos de inovação & $\mathbf{4}$ & $\mathbf{7 , 4 \%}$ \\
\hline Escassez de fontes apropriadas de financiamento & $\mathbf{9}$ & $\mathbf{1 6 , 7 \%}$ \\
\hline Rigidez organizacionais & $\mathbf{8}$ & $\mathbf{1 4 , 8 \%}$ \\
\hline Falta de pessoal qualificado & $\mathbf{5}$ & $\mathbf{9 , 3 \%}$ \\
\hline Falta de informação sobre tecnologia & $\mathbf{8}$ & $\mathbf{1 4 , 8 \%}$ \\
\hline Escassas possibilidades de cooperação com outras & $\mathbf{1 3}$ & $\mathbf{2 4 , 1 \%}$ \\
\hline Dificuldade para se adequar a padrões, normas e regulamentações & $\mathbf{9}$ & $\mathbf{1 6 , 7 \%}$ \\
\hline A fraca resposta dos consumidores quanto a novos produtos & $\mathbf{2 3}$ & $\mathbf{4 2 , 6 \%}$ \\
\hline Centralização da atividade inovadora em outra empresa do grupo & $\mathbf{6}$ & $\mathbf{1 1 , 1 \%}$ \\
\hline
\end{tabular}




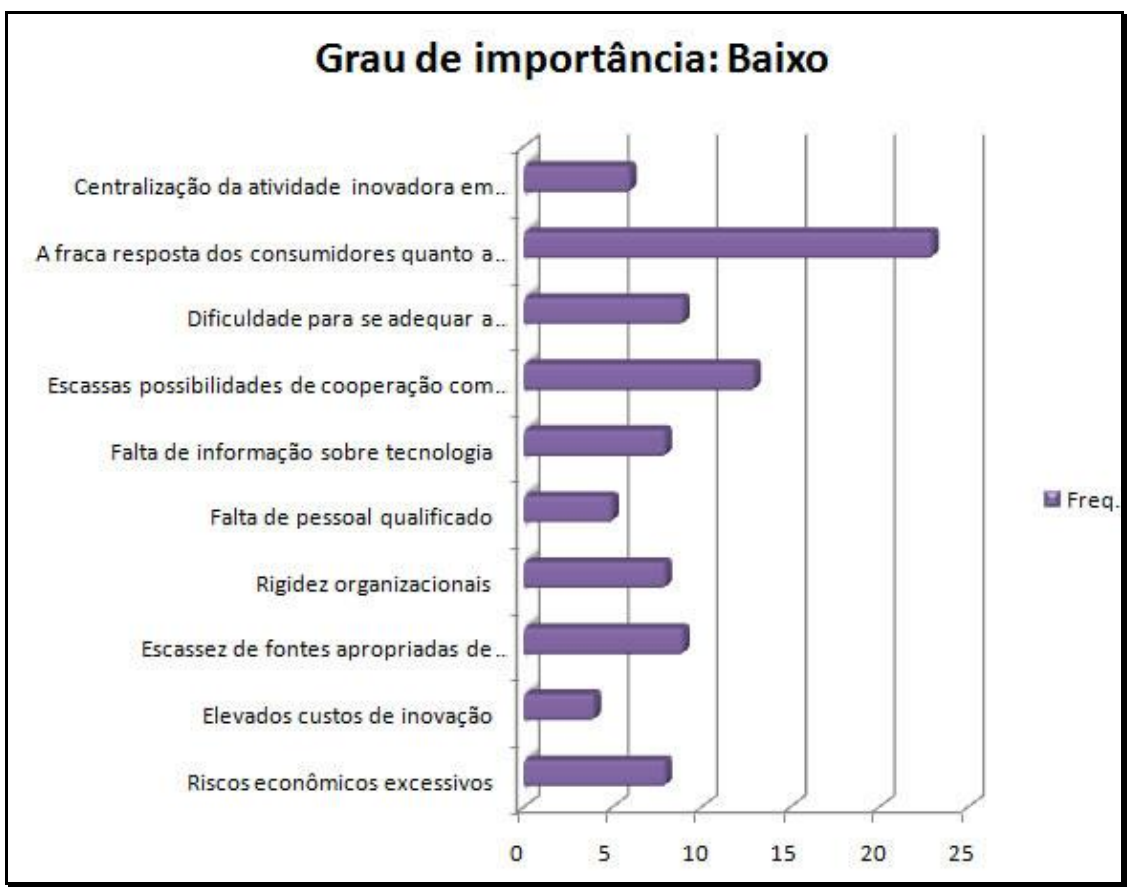

Gráfico 7: Fatores que prejudicaram a inovação das indústrias com grau de importância baixo.

Tabela 6: Fatores que prejudicaram a inovação das indústrias com grau de importância não relevante. Frequência e Porcentagem baseados no total de 54 indústrias.

\begin{tabular}{|l|l|l|}
\hline Grau de importância: Não relevante & Freq. & \% \\
\hline Riscos econômicos excessivos & $\mathbf{6}$ & $\mathbf{1 1 , 1 \%}$ \\
\hline Elevados custos de inovação & $\mathbf{3}$ & $\mathbf{5 , 6 \%}$ \\
\hline Escassez de fontes apropriadas de financiamento & $\mathbf{1 1}$ & $\mathbf{2 0 , 4 \%}$ \\
\hline Rigidez organizacionais & $\mathbf{1 0}$ & $\mathbf{1 8 , 5 \%}$ \\
\hline Falta de pessoal qualificado & $\mathbf{4}$ & $\mathbf{7 , 4 \%}$ \\
\hline Falta de informação sobre tecnologia & $\mathbf{8}$ & $\mathbf{1 4 , 8 \%}$ \\
\hline Escassas possibilidades de cooperação com outras & $\mathbf{7}$ & $\mathbf{1 3 , 0 \%}$ \\
\hline Dificuldade para se adequar a padrões, normas e regulamentações & $\mathbf{7}$ & $\mathbf{1 3 , 0 \%}$ \\
\hline A fraca resposta dos consumidores quanto a novos produtos & $\mathbf{9}$ & $\mathbf{1 6 , 7 \%}$ \\
\hline Centralização da atividade inovadora em outra empresa do grupo & $\mathbf{7}$ & $\mathbf{1 3 , 0 \%}$ \\
\hline
\end{tabular}




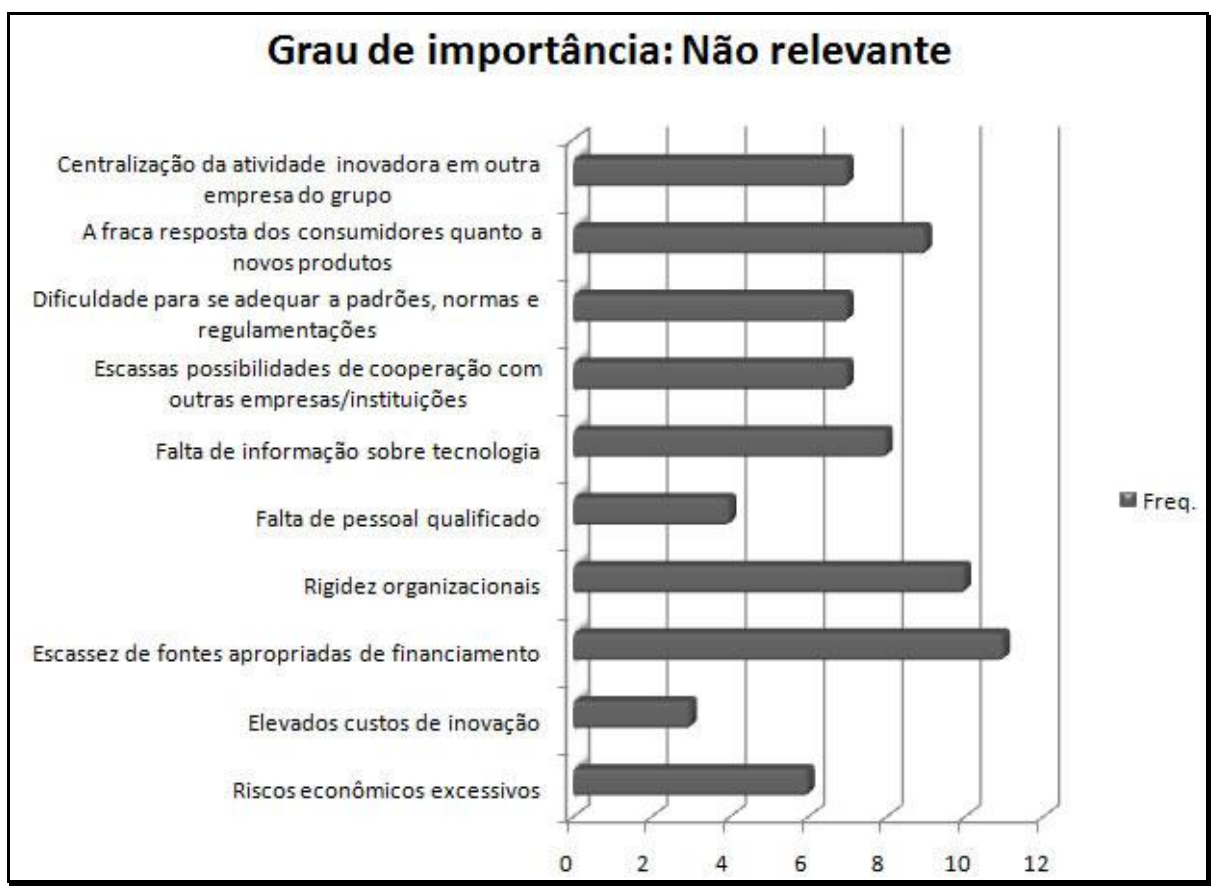

Gráfico 8: Fatores que prejudicaram a inovação das indústrias com grau de importância não relevante.

De acordo com a pesquisa realizada, as dificuldades que mais afetam na inovação, em ordem de importância, são:

- a falta de pessoal qualificado;

- custos elevados;

- riscos econômicos excessivos;

- dificuldade para se adequar a padrões, normas e regulamentações;

- escassas possibilidades de cooperação com outras indústrias/instituições, dentre outros.

Staub (2001) sugere que essas dificuldades ocorrem devido a alguns problemas estruturais do sistema de inovação brasileiro: instabilidade de recursos financeiros federais nas áreas de ciência e tecnologia; baixa participação do setor privado nos investimentos nacionais em P\&D; poucos ou tímidos incentivos fiscais; inadequada estrutura de financiamentos e incentivos às atividades de P\&D.

\section{CONCLUSÕES}

Com base nos resultados obtidos através da pesquisa, observa-se que apesar das dificuldades, as indústrias ainda estão conseguindo implantar inovações em seus produtos e processos, seja com produtos/processos novos ou aperfeiçoados, conseguindo atingir tanto o mercado nacional como o internacional. Buscam-se parcerias com outras empresas e instituições ou até contratação de 
organizações externas para que essas inovações auxiliem no crescimento da empresa, assim como aumentem as possibilidades de competitividade no mercado. As atividades de P\&D são consideradas de importância alta pelas indústrias entrevistadas, porém, a maioria delas não possui um departamento específico devido aos altos custos de implementação, comparado com a quantidade de inovações realizadas.

As indústrias que não realizaram inovações nos últimos quatro anos não justificaram os motivos. Os obstáculos foram sugeridos pela pesquisa, questionando o grau de importância para cada indústria. Observou-se que os pontos mais afetados estavam relacionados à contratação de profissionais e custos de inovação. Ficou claro que as dificuldades enfrentadas principalmente pelas pequenas indústrias para inovar são muitas. Além disso, a inovação envolve uma série de esforços, necessários para que a empresa consiga manter-se no mercado, tornando-se cada vez mais competitiva, capaz de competir com grandes marcas do seu próprio setor.

\section{REFERÊNCIAS}

BACHMANN, D. L.; DESTEFANI, J. H. (2008). Metodologia para Estimar o Grau de Inovação nas MPE: Cultura do Empreendedorismo e Inovação. Recuperado em 04 maio, 2012, de http://www.bachmann.com.br/website/documents/ArtigoGraudeInovacaonasMPE.pdf.

CARON, A. (2004). Inovação tecnológica em pequenas e médias empresas. Revista FAE Business. $\mathrm{n}^{\circ}$ 8, 25-28. Recuperado em 09 maio, 2012, de http://www.unifae.br/publicacoes/pdf/revista_da_fae/ fae_v8_n1/rev_fae_v8_n1_06_prof_caron.pdf.

DESS, G. G.; LUMPKIN, G. T. (2005). The role of entrepreneurial orientation in stimulating effective corporate entrepreneurship. The Academy of Management Executive, 19 (1), 147-156.

FERRAZ, J.C.; KUPFER, D.; HAGUENAUER, L. (1995). Made in Brazil: Desafios Competitivos para a indústria. Rio de Janeiro: Campus.

GAGNON, Y.-C.; TOULOUSE, J.-M. (1996). The Behavior of Business Managers when Adopting New Technologies. Technological Forecasting and Social Change. 52 (1) 59-74.

HAGEDOORN, J. (1994). Schumpeter: an appraisal of his theory of innovation and entrepreneurship. MERIT Research Memorandum, 2/94-020. 
HITT, M. A.; IRELAND, R. D.; HOSKISSON, R. E. (2002). Administração estratégica. São Paulo: Pioneira Thomson Learning.

IBGE. Pesquisa de Inovação: PINTEC - 2011. Recuperado em 09 maio, 2012, de http://www.pintec. ibge.gov.br/downloads/InstrucoesPINTEC2011.pdf.

KOTLER, P. (2000). Administração de marketing: análise, planejamento, implementação e controle. (10a. ed.) São Paulo: Prentice-Hall.

LA ROVERE, R.L. (1999). As Pequenas e Médias Empresas na Economia do Conhecimento: implicações para políticas de inovação. In: LASTRES, H.M.M., e ALBAGLI, S., Informação e Globalização na Era do Conhecimento. Rio de Janeiro: Campus.

MANSFIELD, E. (1978). Microeconomia: teoria e aplicações. Rio de Janeiro, Editora Campus.

MOVIMENTO BRASIL COMPETITIVO (2008). Manual de Inovação. Brasília.

OCDE (2005). Manual de Oslo: diretrizes para a coleta e interpretação de dados sobre inovação tecnológica. Publicado pela FINEP (Financiadora de Estudos e Projetos), $3^{\text {a }}$ edição,.

OCDE (1992). Technology and the Economy: The key relationships, Paris, OCDE.

OECD (1995), Information Technology (IT): Diffusion Policies for Small and Medium-sized Enterprises (SMEs). Recuperado em 09 maio, 2012, de http://www.oecd.org/dsti/sti/it/infosoc/prod/e_ 95-76.pdf. Working paper, vol. 3, GD(95)76, Organisation for Economic Co-operation and Development, Paris.

ROTHWELL, R.; DODGSON, M. (1993). Technology-based SMEs: their role in industrial and economic change, International Journal of Technology Management, Special publication on small firms and innovation, 8-22.

SBRAGIA, R. (2001). Avaliação dos resultados de P\&D na empresa: uma possível abordagem para o problema. In: VASCONCELLOS, Eduardo (Org). Gerenciamento da Tecnologia: um instrumento para a competitividade empresarial. São Paulo: Blücher.

SCHUMPETER, J. A. (1982). Teoria do Desenvolvimento Econômico. São Paulo: Abril Cultural.

STAUB, E. (2001). Desafios estratégicos em ciência, tecnologia e inovação. IEDI. Brasília. Recuperado em 04 maio, 2012, de http://www.iedi.org.br/admin_ori/pdf/desafios.pdf. 
STEFANOVITZ, J.P.; NAGANO, M.S. (2005, novembro). Aquisição e criação de conhecimento na indústria de alta tecnologia. Anais do XXV Encontro Nacional de Engenharia de Produção. Porto Alegre, RS, Brasil, 29.

TETHER, B.S. (2003). What is innovation? approaches to distinguishing new products and services from existing products and processes ESRC. Centre for Research on Innovation and Competition (CRIC), 12, 2-41.

TRAILL, B. (1997). Structural Changes in European food industry: consequences of innovation. In Traill and Grunert (eds). Product and process innovationin the food industry. Chapman and Hall: London.

\title{
PERFORMANCE ANALYSIS OF REGIONAL INNOVATION - A CASE STUDY IN THE INDUSTRY
}

\begin{abstract}
This paper makes an analysis of regional innovation performance, conducted with the aim of adapting from a methodological investigate the level of technological innovation in the industries of a region in four years and the relationship with regional development. We developed a method based on PINTEC/IBGE associated with the "Oslo Manual". A semi-structured survey was administered in 54 industries from various sectors that characterize the contribution to the development of this region. The results showed that despite numerous obstacles, industries are achieving a level of innovative activity in their products and processes, thus improving the market competitiveness and contributing to the development of the region.
\end{abstract}

Keywords: Technological innovation; Innovation management; Oslo manual; Industries.

Data do recebimento do artigo: 15/08/2013

Data do aceite de publicação: 17/03/2014

Revista de Administração e Inovação, São Paulo, v. 11, n.2, p. 71-88, abr./jun. 2014. 